\title{
Label-free bioaffinity detection using terahertz technology
}

\author{
Samuel P Mickan ${ }^{1,3}$, Abdellah Menikh ${ }^{1}$, Haibo Liu ${ }^{1}$, \\ Carmen A Mannella', Robert MacColl' ${ }^{2}$, Derek Abbott ${ }^{3}$, \\ Jesper Munch ${ }^{4}$ and X-C Zhang ${ }^{1}$ \\ ${ }^{1}$ Center for Terahertz Research, Department of Physics, Applied Physics \& Astronomy, \\ Rensselaer Polytechnic Institute, NY 12180, USA \\ 2 Wadsworth Center, ESP-PO Box 509, Albany, NY 12201, USA \\ 3 Centre for Biomedical Engineering, and Department of Electrical \& Electronic Engineering, \\ The University of Adelaide, Adelaide, SA 5005, Australia \\ ${ }^{4}$ Department of Physics \& Mathematical Physics, The University of Adelaide, Adelaide, \\ SA 5005, Australia \\ E-mail: zhangxc@rpi.edu
}

Received 1 March 2002

Published 17 October 2002

Online at stacks.iop.org/PMB/47/3789

\begin{abstract}
We report the first use of differential terahertz time-domain spectroscopy for bioaffinity sensing. Binding is observed by measuring the transmission of a thin layer of biotin bound to the sensor protein avidin. We demonstrate the $\mathrm{THz}$ wave transmission of a sub-micron-thick film and sensitivity to $0.1 \mu \mathrm{g} \mathrm{cm}^{-2}$ of biotin. These results point the way for a host of biosensor applications using T-rays, or pulsed far-infrared (FIR) radiation.
\end{abstract}

\section{Introduction}

In the past decade the development of optical biosensors has mainly been concentrated in the UV-visible and the mid-infrared regions of the electromagnetic spectrum. This is due to the availability of sources, detectors and fibre optics, which have helped develop sensitive, specific, portable biosensors and state-of-the-art remote sensing systems. In contrast, the use of terahertz spectroscopy $\left(1 \mathrm{THz}=10^{12} \mathrm{~Hz}\right)$ for sensing purposes is in its infancy. Although terahertz spectroscopy has witnessed enormous progress within the last few years, applications to biological materials are still limited. Here we report for the first time an experimental investigation of the surface recognition process of the highly specific labelfree biotin-avidin complex, monitored with terahertz time-domain spectroscopy (THz-TDS) (Grischkowsky et al 1990).

The affinity between the vitamin biotin and the egg-white glycoprotein avidin, or the related bacterial protein streptavidin, with $K_{a}=10^{15} \mathrm{M}^{-1}$ is nearly as strong as covalent 
bonding. Extreme denaturing conditions are required to disrupt the complex (Green 1975, Cuatrecasas and Wilchek 1968). The avidin-biotin complex is not dissociated in $9 \mathrm{M}$ urea or $6 \mathrm{M}$ guanidinium hydrochloride, even in the $\mathrm{pH}$ range from 2 to 13 . Kinetic studies of avidinbiotin binding suggest that a diffusion-limited association of the complex and its very slow dissociation result in its high affinity (Chilcoti and Stayton 1995). The highly specific and strong binding of the avidin-biotin system has led to its wide usage in a variety of biochemical and biotechnological applications.

An essential prerequisite in the successful application of biotin-avidin technology is the incorporation of the biotin moiety into biomolecule systems. Fortunately, following biotinylation, the biological activity and the physicochemical properties of the biotin-modified molecules are commonly retained. Another distinctive advantage of this system are the four identical binding sites of avidin for biotin that provide the possibility of cross-linking between different biotin-containing molecules, and add another dimension to the use of this multifaceted system in signal amplification. The importance of the biotin-avidin system has been highlighted in numerous studies using a wide range of biophysical and biochemical techniques (Wilchek and Bayer 1988).

Immobilization of biomolecules on solid surfaces is a key problem in biosensor technology and the biotin-avidin system offers an elegant way to couple macromolecules to supported membranes. Adsorbing avidin on solid surfaces and assembling biotinylated molecules shows that a number of functionally active ligands can be immobilized (O'Shannessy et al 1992, Brink et al 1994). Using this technology makes it possible to attach active ligands to the biosensor surface, thereby identifying target species in the sample, and increasing the selectivity of the biosensor by reducing interferences from non-specific interactions.

For label-free detection, the presence of a bound biotin layer on an avidin film can be measured using a variety of biosensing techniques such as quartz crystal microbalance spectroscopy, ellipsometry and surface plasmon resonance spectroscopy (Minunni and Mascini 1995, Mandenius and Mosbach 1988, Masson et al 1994). Our approach in this study is to present a novel technique based on FIR transmission changes caused by a biotin film binding to an avidin layer. The FIR transmission depends on the refractive index of both target and receptor molecules.

\section{Experimental design}

\subsection{Differential THz-TDS (DTDS)}

DTDS reduces fluctuations in a small T-ray waveform by measuring only the difference between the FIR transmission of two thin film samples, one reference sample and one sample exposed to the target substance. In this case, a pure avidin film acts as a reference to a film of biotin bound to avidin (figure 1). Double-modulated DTDS has been used for characterizing micron-thick films and is described elsewhere in detail (Jiang et al 2000, Brucherseifer et al 2000, Mickan et al 2002a). Figure 2 shows a schematic of a double-modulated DTDS spectrometer. The basic DTDS concept of rapidly switching between a reference and a sample is pictured in figure 3(c). The T-ray waveform measured by DTDS, $y_{\text {dif }}$, is directly proportional to the difference between the reference and sample waveforms, $y_{\text {ref }}-y_{\text {sam }}$. Rapidly switching between the sample and the reference allows an increase in the signal-to-noise ratio (SNR) of the measurements by a factor of approximately $S_{\mathrm{sam}} / S_{\mathrm{dif}}$, where $S$ is the Fourier spectrum of the time-domain waveform $y$ (Mickan et al 2002b). Thus for very small differential signals $S_{\text {dif }}$, there is a marked improvement in SNR. 


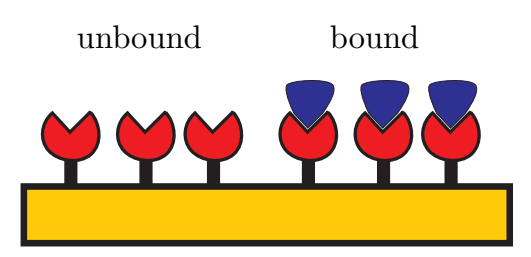

Figure 1. Schematic of bound and unbound avidin molecules on a glass sample slide. The protein avidin is functionalized to the surface of the slide and used as a sensor for biotin molecules. DTDS is used to detect the FIR transmission of the biotin layer bound to the avidin.

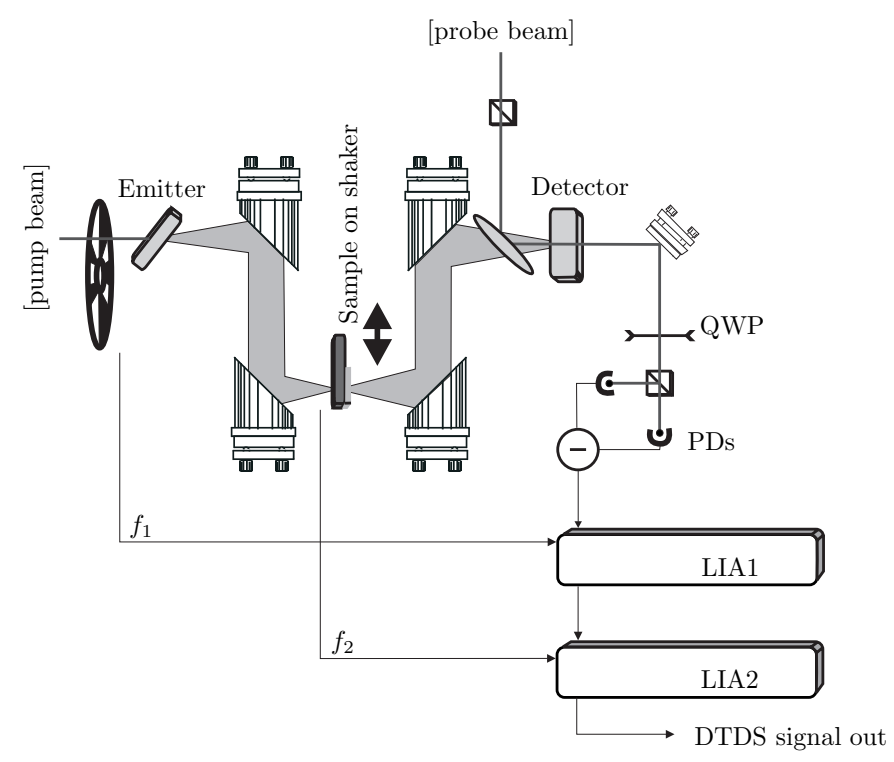

Figure 2. Double-modulated DTDS schematic. The pump and probe beams are split from a femtosecond laser and act to generate and detect the T-rays. The T-rays, shaded in grey, are collimated and focused with gold-plated parabolic mirrors. The T-ray electric field is converted to an electronic signal with the detector crystal, a quarter wave plate (QWP) and balanced photodiodes (PDs). Two lock-in amplifiers (LIAs), with the shaker and an optical modulator, implement the double modulation scheme. The DTDS output signal depends on the differences between the biotin-avidin and pure avidin films (Mickan et al 2002a).

\subsection{Sample slide preparation}

Prior to deposition of the avidin film, the glass slides were cleaned, silanized and crosslinked. Two glass microscope slides, each approximately $0.96 \mathrm{~mm}$ thick were prepared simultaneously, one as the test sample and the second as a reference. The reference slide of pure avidin is used to confirm that the DTDS signal is due entirely to the presence of biotin-avidin binding and not inhomogeneities in the avidin film. The glass substrates were cleaned with $50 \%$ hot nitric acid, then rinsed several times with doubly-distilled water. The substrate was placed in a 5\% solution of 3-mercaptopropyltrimethoxysilane in toluene for $1 \mathrm{~h}$. The organic cross linker succinimide ester was dissolved in dimethylformide to a final concentration of $3 \mathrm{mM}$. The silanized substrate was treated with the cross linker for another hour, washed thoroughly in phosphate-buffered saline (PBS) and dried under vacuum. 


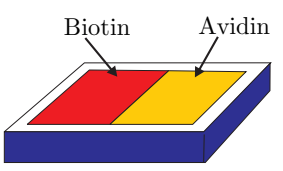

(a)

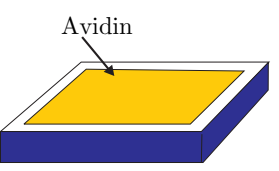

(b)

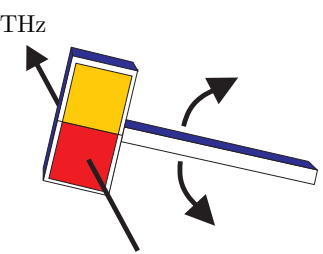

(c)

Figure 3. Biosensor slide configurations used in DTDS. The two slides, (a) biotin-avidin slide and (b) pure avidin test slide, were prepared as detailed in section 2, and mounted for DTDS as in (c).

\subsection{Avidin immobilization}

$2 \mathrm{mg}$ of avidin were dissolved in $10 \mathrm{ml}$ of sodium phosphate buffer, $\mathrm{pH} \mathrm{7.4.} \mathrm{An} \mathrm{aliquot} \mathrm{of}$ $200 \mu \mathrm{l}$ solution was applied to each glass slide, and incubated for about $30 \mathrm{~min}$, producing a film surface area of approximately 15 by $30 \mathrm{~mm}$. After washing and drying under nitrogen, half of the biosensor slide was exposed to $10 \mu \mathrm{l}$ of a solution containing $10 \mu \mathrm{g} \mathrm{ml}^{-1}$ of biotin, dissolved in chloroform-methanol, mixed in the ratio of five to one. Avidin is a very stable protein, and the avidin-biotin complex is known to retain its activity even in strong denaturants (Anzai et al 2000). The resulting concentration of biotin is $0.1 \mu \mathrm{g} \mathrm{cm}^{-2}$. The ensemble was washed with a mixture of PBS to remove unbound molecules. The reference slide of pure avidin was not exposed to the biotin solution.

Washing the slide removes any material that is weakly bound to the avidin layer. Avidin-biotin binding is extremely strong, so non-specifically bound molecules are removed preferentially. The two biosensor slides are shown in figure 3. The biosensor film thickness is estimated to be approximately $1 \mu \mathrm{m}$, which corresponds to many layers of the protein avidin. The thickness of the biotin layer is approximately $40 \mathrm{~nm}$, as measured with an atomic force microscope (AFM). The topographical roughness of avidin film both with and without biotin, measured on the AFM, is of the same order of magnitude, and randomly distributed across the surface with a quasi-period of about $30 \mu \mathrm{m}$. When measured with DTDS, the signal is a spatial average of the spot size of the focused T-ray beam, which has a $1 \mathrm{~mm}$ diameter (FWHM). The random roughness of the film is largely averaged out over the T-ray spot, while the consistent contribution of the biotin in half of the biosensor generates a discernable signal.

\subsection{Experimental conditions}

Our T-ray system has been described elsewhere (Mickan et al 2002a). The femtosecond laser source generates 150 fs pulses at $86 \mathrm{MHz}$ and $1.5 \mathrm{~W}$ of average power. The pump beam is $700 \mathrm{~mW}$ after the optical modulator, and T-rays are generated in an unbiased GaAs wafer. The T-ray detector is a 2-mm-thick crystal of $\langle 110\rangle \mathrm{ZnTe}$. The experiment is conducted at room temperature and about $40 \%$ relative humidity. Our T-ray waveforms are sampled with a $15 \mathrm{THz}$ bandwidth and a $23 \mathrm{GHz}$ spectral resolution. The sample is mounted in a galvanometer in the T-ray beam and modulated at $10 \mathrm{~Hz}$ over a peak-to-peak distance of more than $10 \mathrm{~mm}$. The T-ray beam is double-modulated with an optical modulator at $2 \mathrm{kHz}$ and the lock-in amplifier time constant was set to $1 \mathrm{~s}$. 


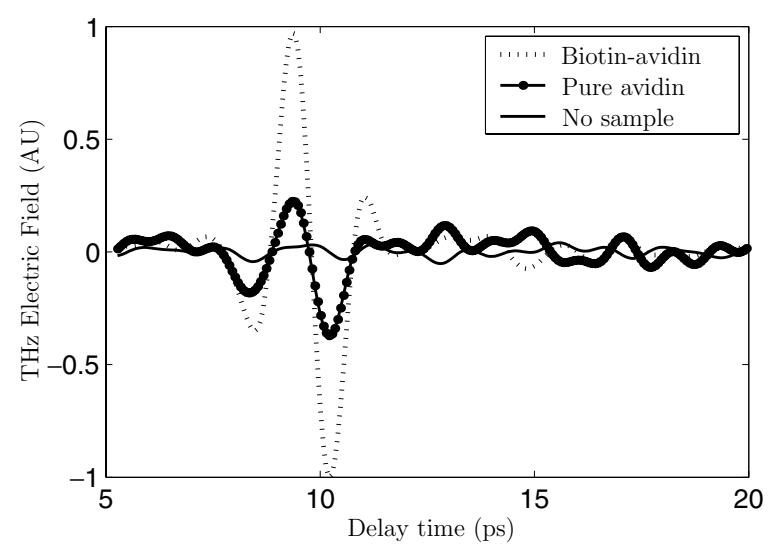

Figure 4. Time-domain differential T-ray pulses (output from LIA2). These waveforms have been normalized to the peak of the biotin-avidin waveform and smoothed using a $0.15-0.95 \mathrm{THz}$ band-pass filter. The three cases shown are (1) the signal from the biotin-avidin interface, (2) noise from topographic variations in the pure avidin film and (3) inherent noise in the DTDS system 'no sample'. The variability in the biotin-avidin over multiple scans is less than the thickness of the plotted line. Each waveform shown was measured with a time constant of $1 \mathrm{~s}$ on LIA2.

\section{Results}

\subsection{DTDS of biotin-avidin}

The time-domain differential results are shown in figure 4. There is a clear differential response from the biotin-avidin film.

Figure 4 shows as a dotted line the time-domain waveforms from two experiments on different lateral sections of the same biotin-avidin sample. This gives an indication of the SNR, the repeatability of the experiment and the homogeneity of the film. Although there is some variability in these results, there is a clear differential signal, indicating differences in the THz properties of biotin-avidin and a pure avidin film. The waveforms have been filtered in the spectral domain to attenuate frequencies where the T-ray signal is weak.

The bound biotin molecules form a second film approximately $40 \mathrm{~nm}$ thick, as measured in section 2.3. The additional thin film of biotin causes a change in the FIR properties of the biosensor, which is detected by DTDS. Washing with PBS was used to remove non-biotin molecules that may have weakly attached to the avidin sensor layer. We will confirm that our signal is not due to other non-specifically bound molecules in future work. The specificity can be explored by applying a solution of non-biotin biomolecules to the test slide, washing with PBS, and measuring the DTDS signal.

\subsection{DTDS of pure avidin}

We perform a null test of the DTDS system with a film of pure avidin. This demonstrates that the signal in section 3.1 is due primarily to the presence of biotin and not to inhomogeneities in the avidin film. The pure avidin sample is mounted in exactly the same manner as the biotin-avidin sample. The time-domain DTDS waveform of the pure avidin film is shown as a solid line with a dot in figure 4. The avidin-avidin signal is less than half the biotin-avidin signal. Sample inhomogeneities are due to the sample preparation and can be reduced by more uniform deposition techniques. 


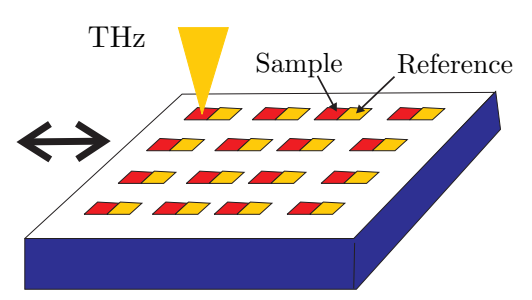

Figure 5. Array of biosensors for DTDS.

\subsection{DTDS with no sample}

The noise inherent in double-modulated DTDS can be measured by completely removing the galvanometric modulator from the T-ray beam and monitoring the DTDS signal. Since the beam is no longer being modulated at $f_{2}$, as in figure 2 , there should be no signal. Any measurement is due to noise in the T-ray system, primarily from pump laser instability (von der Linde 1986), frequency instability of the modulators and spectral leakage in the second lock-in amplifier, LIA2. The standard, as opposed to differential, T-ray pulse is rejected by the bandwidth of the low-pass filter in LIA2. The standard pulse rejection is improved by using a higher sample modulation frequency $f_{2}$ and a longer LIA 2 time constant. For example, the standard pulse rejection is improved five-fold by modulating the sample at $10 \mathrm{~Hz}$ instead of $5 \mathrm{~Hz}$. The limitations on $f_{2}$ are travel and heating of the galvanometer, and the trade-off with increasing time constant is a longer waveform acquisition time.

\section{Conclusions and future work}

In this study we have demonstrated for the first time the use of T-rays for label-free biosensing of biotin-avidin binding. A detection limit of $0.1 \mu \mathrm{g} \mathrm{cm}^{-2}$ has been achieved, which is comparable to established biosensor technologies in reflectometry and ellipsometry (Mandenius and Mosbach 1988). The sensitivity of the T-ray sensor seems to depend on the immobilization chemistry of avidin on the glass slide, the analyte-ligand binding affinity, the homogeneity of film deposition, the THz refractive index mismatch between the sensor and bound layers, and the instrument SNR. This study reveals the potential of T-rays to monitor the recognition process at the low detection limit of biomolecules. In future work, we will determine the limits of our sensitivity to thin layers of bound biomolecules and estimate the possibility of noise caused by non-specific binding. We will explore new potential targets, trace gas sensing, antigen-antibody binding and array development, as conceptualized in figure 5 .

\section{Acknowledgments}

We would like to acknowledge the support of the US Army Research Office, and the US National Science Foundation, the Fulbright Commission, and the Australian Research Council. We would like to thank Deli Liu at RPI for assistance with the AFM.

\section{References}

Anzai J I, Hoshi T and Osa T 2000 Avidin-biotin mediated biosensors Biosensors and Their Applications ed V C Yang and T T Ngo (New York: Academic) pp 35-46 
Brink G, Schmitt L, Tampe R and Sackmann E 1994 Self assembly of covalently anchored phospholipid supported membranes by use of DODA-Suc-NHS-lipids Biochim. Biophys. Acta 1196 227-30

Brucherseifer M, Pellemans H P M, Haring Bolívar P and Kurz H 2000 THz spectroscopy with ultrahigh sensitivity Conf. on Lasers and Electro-Optics '00, vol 39 of TOPS (Optical Society of America, San Francisco, CA, USA) pp 553-4

Chilcoti A and Stayton P S 1995 Molecular origin of the slow streptavidin-biotin dissociation kinetics J. Am. Chem. Soc. 117 10622-8

Cuatrecasas P and Wilchek M 1968 Single-step purification of avidin from egg white by affinity chromatography on biocytin-sepharose columns Biochem. Biophys. Res. Commun. 33 235-9

Green N M 1975 Avidin Adv. Prot. Chem. 29 85-133

Grischkowsky D, Keiding S, van Exter M and Fattinger C 1990 Far-infrared time-domain spectroscopy with terahertz beams of dielectrics and semiconductors J. Opt. Soc. Am. B: Opt. Phys. 7 2006-15

Jiang Z, Li M and Zhang X-C 2000 Dielectric constant measurement of thin films by differential time-domain spectroscopy Appl. Phys. Lett. 76 3221-3

Mandenius C F and Mosbach K 1988 Detection of biospecific interactions using amplified ellipsometry Anal. Biochem. $17068-72$

Masson L, Mazza A and Brousseau R 1994 Stable immobilization of lipid vesicles for kinetic studies using surface plasmon resonance Rev. Anal. Biochem. 218 405-12

Mickan S P, Lee K S, Lu T-M, Munch J, Abbott D and Zhang X-C 2002a Double modulated differential THz-TDS for thin film dielectric characterization Microelectr. $J$. accepted

Mickan S P, Abbott D, Munch J and Zhang X C 2002b Noise reduction in terahertz thin film measurements using a double modulated differential technique Fluctuat. Noise Lett. 2 13-29

Minunni M and Mascini M 1995 The quartz crystal microbalance as biosensor: a status report on its future Anal. Lett. 28 749-64

O'Shannessy D J, Burker M B and Peck K 1992 Immobilzation chemistries suitable for use in the BIAcore surface plasmon resonance detector Anal. Biochem. 205 132-6

von der Linde D 1986 Characterization of the noise in continuously operating mode-locked lasers Appl. Phys. B: Photophys. Laser Chem. 39 201-17

Wilchek M and Bayer E A 1988 The avidin-biotin complex and bioanalytical applications Anal. Biochem. 171 1-32 